\title{
Heart and musculoskeletal hemodynamic responses to repetitive bouts of
}

quadriceps static stretching

3

Massimo Venturelli ${ }^{1,2,3}$, Susanna Rampichini ${ }^{1}$, Giuseppe Coratella ${ }^{1}$, Eloisa Limonta ${ }^{1,4}$, Angela Valentina Bisconti ${ }^{1,3}$, Emiliano Cè ${ }^{1,4}$, Fabio Esposito $^{1,4}$.

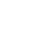

${ }^{1}$ Department of Biomedical Sciences for Health, Università degli Studi di Milano, via G.

Colombo 71, 20133 Milan, Italy;

${ }^{2}$ Department of Neurosciences, Biomedicine and Movement Sciences. University of Verona.

Via Casorati 43, 37131 Verona, Italy;

${ }^{3}$ Department of Internal Medicine section of Geriatrics, University of Utah, Salt Lake City, UT, USA.

${ }^{4}$ IRCCS Istituto Ortopedico Galeazzi. Via Riccardo Galeazzi, 4, 20161 Milan, Italy;

.

Short running head: hemodynamic response to muscle stretching

Author Contributions: Dr. Venturelli and Esposito had full access to all of the data in the study and take responsibility for the integrity of the data and the accuracy of the data analysis. Design and Conduct of Study: Venturelli, Cè, Rampichini, Esposito. Collection, analysis, and data interpretation: Venturelli, Cè, Coratella, Bisconti, Limonta, Rampichini. Review and final approval of manuscript: Venturelli, Cè, Coratella, Limonta, Bisconti, Rampichini, Esposito.

\section{Corresponding author:}

Massimo Venturelli, $\mathrm{PhD}$

Department of Neurosciences, Biomedicine and Movement Sciences. University of Verona.

Via Casorati 43, 37131 Verona, Italy;

e-mail: massimo.venturelli@univr.it

orcid.org/0000-0002-2469-8787 
Abstract (250 w)

The role of sympathetic and parasympathetic activity in relation to the repetitive exposure to static stretching (SS) on heart and musculoskeletal hemodynamics in stretched and resting muscles is still a matter of debate. The aim of the study was to determine cardiac and musculoskeletal hemodynamics to repetitive-bouts of unilateral SS. Sympathetic and parasympathetic activity contribution to the central hemodynamics and local difference in circulation of stretched and resting muscles were also investigated. In eight participants, heart rate (HR), cardiac output (CO), mean arterial pressure (MAP), HR variability (HRV), blood pressure variability (BPV), blood flow in passively stretched limb (SL) and control (CL, resting limb) were measured during 5 bouts of unilateral SS (45s of knee flexion and $15 \mathrm{~s}$ of knee extension). SS increased sympathetic $(\approx 20 \%)$ and decreased parasympathetic activity $(\approx 30 \%)$ with a prevalence of parasympathetic withdrawal. During SS, HR, CO, and MAP increased by $\approx 18 \mathrm{BPM}, \approx 0.29 \mathrm{l} \cdot \mathrm{min}^{-1}, \approx 12 \mathrm{mmHg}$, respectively. Peak blood flow in response to the $1^{\text {st }}$ stretching maneuver increased significantly $\left(+377 \pm 95 \mathrm{ml} \cdot \mathrm{min}^{-1}\right)$ in the $\mathrm{SL}$, and reduced significantly $\left(-57 \pm 48 \mathrm{ml} \cdot \mathrm{min}^{-1}\right)$ in the CL. This between-limb difference in local circulation response to SS disappeared after the $2^{\text {nd }}$ SS bout. These results indicate that heart hemodynamic responses to SS are primarily influenced by the parasympathetic withdrawal rather than by the increase in sympathetic activity. The balance between neural and local factors contributing to blood flow regulation was affected by the level of SS exposure, likely associated with differences in the bioavailability of local vasoactive factors throughout the stretching bouts. 
56 Repetitive-exposure to static stretching (SS) on heart and musculoskeletal hemodynamics in 57 stretched and remote muscles may be influenced by neural and local factors. We documented 58 that SS-induced heart hemodynamic responses are primarily influenced by parasympathetic 59 withdrawal. The balance between neural and local factors contributing to the regulation of 60 musculoskeletal hemodynamics is dependent on SS exposure possibly because of different 61 local vasoactive factors bioavailability during the subsequent stretching bouts.

62

63 Keywords

64 Hemodynamics, stretching, sympathetic activity, parasympathetic activity

65

66 Glossary

67 SS, passive static stretching; FL, flexion phase; EX, extension phase; FBF, femoral blood 68 flow; SL, stretched leg; CL control resting leg; HR heart rate; CO, cardiac output; SV, stroke 69 volume; MAP, mean arterial pressure; VC, vascular conductance; HRV, heart rate variability; 70 RMSSD, root mean square of the squared differences of successive RR intervals; LF, low 71 frequency; HF, high frequency; BPV, blood pressure variability; SBP, systolic blood 72 pressure; DBP diastolic blood pressure; $\Delta$ peak, relative changes; AUC, area under the curve. 


\section{Introduction}

The control of musculoskeletal blood flow is a complex integrative mechanism that equalizes the vasoconstrictive and vasodilatory triggers to distribute blood flow within and between skeletal muscles $(6,36)$. The homeostasis of systemic (neural) and local factors is crucial for the control of skeletal muscle blood flow, with an important factor being the balance between sympathetically-mediated vasoconstriction and the vasodilation induced by local factors, such as nitric oxide (NO) (15). At rest, the equilibrium between vasoconstriction and vasodilation results in a smooth muscle tone near $60 \%$ of the total resistance vessel vasodilatory capacity (39). However, when this balance is altered by the increases in muscle sympathetic nerve activity, the vasculature of resting skeletal muscles can achieve elevated levels of vasoconstriction (39).

During physical exercise, general agreement exists on the key role of the autonomic nervous system in the response to voluntary skeletal muscle activation (30). Indeed, sympathetic and parasympathetic activity are modulated, at least in part, by the parallel activation of the central motor pathways and the feedback that arises from mechano- and metabo-receptor activation in the skeletal muscle $(1,10,16,42)$. Differently to physical exercise, static stretching (SS) is characterized by the absence of exercise-induced increase in muscle metabolism and the lack of central command, both of which would generate a sympathetic-mediated increase in heart rate. Hence, the increase in HR observed during SS has been attributed to a decrease in vagal activity and the concomitant small rise in sympathetic discharge (9). However, whether or not cardiac output (CO), stroke volume (SV), and mean arterial pressure (MAP) response to passive SS is primarily influenced by the sympathetic-mediated activation or by the parasympathetic withdrawal is still poorly understood (30).

The acute physiological effects of passive SS on the above-mentioned heart and musculoskeletal hemodynamics have been recently debated $(18,19,42)$, documenting either 
no detectable change in net blood flow, MAP, and popliteal artery vascular conductance (VC)

$102(18,19)$ or an increase in heart rate $(\mathrm{HR})$ and $\mathrm{CO}$, coupled with a hyperemia to the stretched

103 skeletal muscles (42). The latter observation has been likely explained by the mechano-reflex

104 activation (42) and local nitric oxide (NO) release $(29,37)$. Other seminal studies on this

105 subjects issue, though, reported that the HR and blood pressure responses to calf muscle

106 stretch are independent of the metabo-reflex activation (10) and can decrease spontaneous

107 baroreflex sensitivity and other indexes of vagal tone (7). However, the mechanisms

108 underpinning the contribution of local (vasodilatory) and systemic (vasoconstrictive) factors

109 to these physiological changes and their transitory nature $(\sim 35 \mathrm{~s})$ are not completely

110 understood. Further insights on this phenomenon may come from a new approach involving

111 repetitive exposures to SS. Given the generally low bioavailability of NO as a local

112 vasodilator factor (22), several bouts of SS should deplete temporarily the NO reserve and

113 blunt the SS-induced hyperemia. Moreover, whether or not the mechano-reflex induced

114 increases in sympathetic activity may differently affects the circulation of stretched and

115 remote muscles not directly involved in SS is not clear.

116 Therefore, the aim of the present study was to compare the heart and musculoskeletal

117 hemodynamic responses to repetitive bouts of unilateral quadriceps muscle SS. The

118 contribution of sympathetic and parasympathetic activity to the heart hemodynamics was also

119 investigated. Specifically, by studying HRV, heart hemodynamics, blood pressure variability

120 (BPV), blood flow in passively stretch limb (SL), and control (CL, resting limb) during 5

121 bouts of unilateral SS (45 s of knee flexion and $15 \mathrm{~s}$ of knee extension), we tested the

122 following hypotheses: (i) the heart hemodynamic responses to SS might be primarily

123 influenced by the parasympathetic withdrawal rather than by the increase in sympathetic

124 activity; (ii) the blood flow response to SS in the passively stretched limb would be initially

125 greater in comparison to the contralateral limb; and (iii) this regional difference in peripheral 
126 circulation response to SS would be dependent on the repetitive exposures to SS (number of

127 bouts) that are administered to the investigated muscle.

\section{$130 \quad$ Methods}

131 Participants: Eight young healthy men (age: $25 \pm 2$ yrs; body mass: $71 \pm 8$ kg; stature: $1.80 \pm$

$1320.06 \mathrm{~m}$; mean \pm standard deviation) participated in this study. None of the participants were

133 smokers and all were moderate physically active. All procedures conformed to the standards

134 set by the Declaration of Helsinki and were approved by the ethical committee of the

135 University of Milan. The participants gave written informed consent prior to their

136 participation after full explanation of the purpose of the study and of the experimental

137 procedures. The participants reported to the laboratory in the morning ( 9 - $10 \mathrm{AM})$ in a fasted

138 state. They were asked to abstain from consuming caffeine 24 hours prior the test, and to

139 report to the laboratory without any form of physical exercise of heavy intensity in the

140 previous 48 hours.

142 Experimental design: After a first visit for familiarization purpose, the participants reported

143 to the laboratory a second time, during which single leg SS was performed. All the 144 experimental procedures, from which experimental data were collected, were accomplished 145 during the second visit.

147 Static stretching (SS): The participants rested in a supine position for 20 minutes before 148 starting the data collection and remained in this position throughout the entire duration of the

149 data collection (Figure 1). As previously reported, SS protocol consisted of 5 minutes of 150 resting baseline followed by passive static knee flexion for $45 \mathrm{~s}$ and passive knee extension 151 for $15 \mathrm{~s}$, repeated five times $(5,20,21)$. During the entire SS protocol, knee extensors were 
152 stretched by the same operator up to a point of discomfort lower than 2. This cut-off of

153 discomfort level was chosen in the current investigation in order to minimize the activation of

154 peripheral pain pathways that might interact with group III and IV afference feedback and

155 potentially accentuate the central hemodynamic response (1). The level of discomfort was

156 assessed by a $0-10$ visual analogue scale, being $0=$ no discomfort at all and $10=$ maximum

157 discomfort (25). The knee joint angle was continuously recorded using a dual-axial

158 goniometer (mod. TSD 130A, Biopac System, CA, USA). Force output between the passively

159 stretched leg and the operator arms was recorded during the protocol by a load cell (model

160 SM-2000 N, Interface, Crowthorne, UK). Specifically, the load cell was positioned $5 \mathrm{~cm}$

161 above the ankle of the passively stretched leg and a member of the research team pushed

162 perpendicularly the load cell in order to stretch the leg extensor for 45 seconds (Figure 1). The

163 mean force output during the 45 seconds of the consecutive FL of the SS protocol was than

164 recorded.

165

166 Central hemodynamics: HR, SV, CO, and MAP were determined on a beat-by-beat basis

167 using a finger photoplethysmography device (FinometerPro Finapres Medical Systems,

168 Amsterdam, The Netherlands). The photoplethysmographic cuff was placed on the third

169 finger of the left hand. The height adjustment sensor and reference were positioned following

170 the manufacturer's instructions. The blood pressure signal was calibrated in accordance to the

171 procedure indicated by the manufacturer. SV was estimated using the Modelflow algorithm

172 (Beatscope version 1.1a; Finapres Medical Systems) (4). CO was then calculated as the

173 product of $\mathrm{HR}$ and SV. The same method has been documented to accurately track CO during

174 exercise $(2,35)$, and, as reported in previous investigations, the absolute changes from rest

175 values have been demonstrated to be accurate $(38,40,41)$.

176 
177 Femoral blood flow (FBF): The measurements of arterial blood velocity and vessel diameter

178 were performed in the passively stretched leg (SL) and control resting leg (CL), distal to the

179 inguinal ligament and proximal to the deep, superficial femoral bifurcation with two Logiq

180 S7pro ultrasound systems (General Electric Medical Systems, Milwaukee, WI, USA). The

181 systems were equipped with $12-14 \mathrm{MHz}$ linear array transducers. The common femoral artery

182 diameters were determined along the central axis of the scanned areas. The blood velocity $(v)$

183 was measured using the same probe at a frequency of $5 \mathrm{MHz}$. The measurements of $v$ were

184 obtained second-by-second with the probe positioned to maintain an insonation angle of $60^{\circ}$

185 or less and the sample volume were centered and maximized according to vessels size. After

186 arterial diameter and mean $v\left(v_{\text {mean }}\right)$ assessment, FBF was automatically calculated using the

187 Logiq S7pro software as:

188

$$
F B F=v_{\text {mean }} \cdot \pi \cdot\left(\frac{\text { vessel diameter }}{2}\right)^{2} \cdot 60
$$

where FBF is in milliliters per minute. All scanning and blinded analyses were performed by experienced and skilled sonographers. To account for potential differences in MAP, VC was calculated as: FBF/MAP.

Heart rate variability (HRV): The computer analysis of spontaneous HR and inter-beat

195 intervals oscillation in consecutive cardiac cycles has been recognized to be a credible

196 quantitative marker to assess the activity of the sympathetic and parasympathetic branches of 197 the autonomic nervous system (8). Several indexes have been developed, in both the time and 198 frequency domain, in order to characterize the contribution of the vagal and the sympathetic 199 efferent activity to the cardiovascular control. In the time domain, the root mean square of the 200 squared differences of successive RR (RMSSD) estimates short term variation of HR (8), thus 201 detecting high frequency oscillations caused by parasympathetic activity. In the frequency 
202 domain analysis, the variance of the signal, namely the distribution of power as a function of 203 frequency (power spectral density, PSD), is calculated by means of short fast Fourier 204 transform and, according to the frequency bands classification proposed by the HRV Task 205 Force (8), it is divided in three components: very low frequency, low frequency (LF) and high 206 frequency (HF). The very low frequency component $(\leq 0.04 \mathrm{~Hz})$ is not usually considered in 207 short recordings (5 minutes). Power component of LF band (0.04-0.15 Hz) includes 208 sympathetic as well as parasympathetic influences, while $\mathrm{HF}(0.15-0.4 \mathrm{~Hz})$ band is mainly 209 influenced by the efferent activity of the vagal tone (8). Both markers could be measured in 210 absolute units of power $\left(\mathrm{ms}^{2}\right)$ and in normalized units (n.u.). While the former provides 211 information about the total power of the band, the latter allows to assess the fractional 212 contribution to HR oscillation given by the two bands (LF and HF), excluding the very low 213 frequency component (43). Therefore, the ratio between normalized LF and HF (LF/HF) is 214 computed as an index of the sympatho-vagal balance (3).

215 During this study HR was derived from the electrocardiographic signal (ECG) collected by 216 the photoplethysmography device at $500 \mathrm{~Hz}$. R-peaks of each QRS complex from the 217 continuous ECG signals were detected by a derivative-threshold algorithm. The inter-beat 218 interval series (R-R interval tachogram) was obtained as the difference between the 219 occurrence times of consecutive R-peaks. An expert operator checked the signal and, in case 220 of ectopic beats, the RR series were corrected through a cubic spline interpolation (28). The 221 time domain and frequency domain analysis of the R-R series were conducted considering 5 222 minutes of signal for each condition (Rest Vs Stretching) (8). In view of the frequency 223 domain analysis, the unevenly time-sampled tachogram was interpolated at $4 \mathrm{~Hz}$ by a cubic 224 spline function and successively down sampled at $1 \mathrm{~Hz}$. The PSD, was calculated by means of 225 short fast Fourier transform and the normalized LF and HF bands were subsequently obtained 226 to compute $\mathrm{LF} / \mathrm{HF}$ values. The very-low-frequency component requiring longer data series 227 was not addressed in the present study. 
The cardiac sympatho-vagal balance was obtained by the LF/HF index while variation in

229 parasympathetic activity was estimated, from the time domain analysis, by the RMSSD.

Blood pressure variability (BPV): Blood pressure measurement, if collected concurrently with HR, is known to allow the simultaneous assessment of markers of efferent sympathetic vascular modulation (31). In light of this, the beat-by-beat systolic blood pressure (SBP) and diastolic blood pressure (DBP) series were obtained from the continuous blood pressure signal to characterize blood pressure oscillations (blood pressure variability, BPV). By the

236 previously described photoplethysmography approach, SBP and DBP were measured for 5 minutes during baseline condition, and 5 minutes during the SS procedure. SBP series was composed by the maximum of BP in each RR interval, while the DBP series was made by the minimum of BP following each SBP detection. Being DBP changes negatively related to muscle sympathetic nerve activity burst incidence $(23,34)$, changes in the mean values of DBP series were computed and considered as an index of the (vessels) sympathetic activity.

242 Moreover, as like HRV analysis, also SBP series can be evaluated in the frequency domain therefore being as oscillation in the LF component of the SBP power spectral density $\left(\mathrm{LF}_{\mathrm{SBP}}\right)$

244 associated with an increase in the sympathetic drive (32), it was used as an additional BPV 245 marker of sympathetic activity (11).

Data collection and analysis: SV, CO, MAP, ECG, and knee joint angle and force output 248 underwent A/D conversion system (mod. UM150, Biopac System, Santa Barbara, CA, USA) and were simultaneously acquired $(1000 \mathrm{~Hz})$ by commercially available data acquisition 250 software (AcqKnowledge 4.2, Biopac Systems, Goleta, CA, USA). The software allowed 251 beat-by-beat analysis of $\mathrm{HR}, \mathrm{SV}, \mathrm{CO}$ and MAP throughout the experimental protocols. $v_{\text {mean }}$ 252 was analyzed with $1 \mathrm{~Hz}$ resolution on the Doppler ultrasound systems (GE Logiq S7pro) for $253 \quad 30 \mathrm{~s}$ at rest and during the 5 minute of repetitive single leg SS. From the velocity and femoral 
artery diameter, net FBF was calculated on a second-by-second basis. Prior to analysis, all

255 hemodynamic data were smoothed using a $3 \mathrm{~s}$ rolling average. As the response to passive

256

257

258

259

260

261

262

263

264

265

266

267

268

269

270

271

272

273

274

275

276

277

278

279

stretching is transient and vary between individuals, a peak response was determined for all variables on an individual basis. Maximal absolute (Peak), relative changes ( $\Delta$ peak) and the area under the curve (AUC) were determined for each subject in all measured variables.

Statistical analysis: Raw data were analysed using a statistical software package (IBM SPSS Statistics v. 22, Armonk, NY, USA). In light of a previous article of our group (42), where a difference of about $15 \%$ in femoral blood flow (main outcome) was observed under SS, a sample size of eight participants was selected to ensure a statistical power higher than 0.80 with a type 1 error $<0.05$. To check the normal distribution of the parameters, a Shapiro-Wilk test was applied. Student's t-test was utilized to determine potential differences between baseline and passive SS measurements in the HRV and BPV normally distributed data. A two-way ANOVA for repeated measures [time (6 levels: baseline +5 stretching bout) $\mathrm{x}$ limb (2 levels: stretched (SL) and control limb (CL))] was used to establish differences among conditions for peripheral hemodynamic data. A two-way ANOVA for repeated measures [time (6 levels: baseline +5 stretching bout) $x$ knee joint position (2 levels: flexion (FL) and extension $(E X))]$ was used to establish differences among conditions for central hemodynamic data. A one-way ANOVA [time (5 stretching bouts)] was used to establish differences for ROM and FO. A Tukey's post hoc test was applied to define the location of the difference, when necessary. If Shapiro-Wilk test did not disclose a normal distribution, for central hemodynamic, knee-joint angle of stretched limb, and the force output during the repetitive bouts of SS, a repeated measure ANOVA on ranks test was applied. A WilcoxonSigned Rank test was conversely applied whereby HRV and BPV variables failed the normality test. The level of significance was set at $\alpha<0.05$. Unless otherwise stated, data are presented as mean \pm standard error of the mean. 
Results

All the participants took part in this experimental protocol without reporting discomfort during the stretching procedures. On a scale from 0 to 10 , the average discomfort across all 5 stretch cycles on the passively stretched leg was $1.4 \pm 1.1$ and did not differ among the repetitive bouts of SS $1.3 \pm 0.9,1.6 \pm 0.9,1.6 \pm 1.3,1.7 \pm 1.1$ and $1.5 \pm 1.2$ during $1^{\text {st }}, 2^{\text {nd }}$, $3^{\text {rd }}, 4^{\text {th }}$ and $5^{\text {th }}$ FL respectively.

Heart rate variability (HRV) response to passive static stretching: the effect of 5 consecutive bouts of one leg SS on HRV indexes is summarized in Figure 2 (panel A and B). After the passive SS, RMSSD significantly dropped by $\sim 20 \%(\mathrm{p}=0.041$, Figure 2 , panel A), whereas the LF/HF index significantly increased by $\sim 63 \% \mathrm{p}=0.039$; (Figure 2, panel B).

Blood pressure and BPV response to passive static stretching: After 5 consecutive bouts of

294 one leg SS, the Wilcoxon Signed Rank Test found a significant increase in mean DBP values $295\left(\sim 13 \%, \mathrm{p}=0.008\right.$; Figure 2, panel C) whereas no changes occurred in $\mathrm{LF}_{\text {SBP }}$ (Figure 2, panel 296 D).

Central hemodynamics during flexion (FL) and extension (EX) phases of consecutive bouts

of one leg static stretching: All central hemodynamic values during 5 consecutive bouts of 300 one leg SS are summarized in Table 1 and Figure 3. ANOVA disclosed significant main 301 effects in MAP for Time $(\mathrm{F}=42.7 ; P<0.001)$ and knee-joint position $(\mathrm{F}=1702 ; P<0.001)$, 302 as well as a time $\mathrm{x}$ knee joint position interaction $(\mathrm{F}=135 ; P<0.001)$. Similarly, main effects 303 for Time $(\mathrm{F}=59.1 ; P<0.001)$ and knee-joint position $(\mathrm{F}=62.7 ; P<0.001)$ and time $\mathrm{x}$ knee 304 joint position interaction $(\mathrm{F}=3.1 ; \mathrm{p}=0.012)$ were retrieved in $\mathrm{SV}$. In HR no main effect for 305 time was found $(\mathrm{F}=0.39 ; \mathrm{p}=0.85)$, while there was a main effect for factor knee-joint 
position $(\mathrm{F}=1266 ; \mathrm{p}<0.001)$, and an interaction between time and knee joint position $(\mathrm{F}=$

307 44.4; $p<0.001)$. Similarly, in $\mathrm{CO}$ no main effect $\mathrm{CO}$ for time was retrieved $(\mathrm{F}=0.98 ; \mathrm{p}=$

308 0.43), while a main effect for knee-joint position $(F=29.6 ; p<0.001)$ and a time $x$ knee joint 309 position $(\mathrm{F}=2.4 ; \mathrm{p}=0.046)$ were found. During the $1^{\text {st }} \mathrm{FL}$ procedure MAP was initially

310 increased by $5 \%(\mathrm{p}<0.05$; Figure 3, panel A). This transitory MAP increase was followed by 311 a significant drop $8 \%(\mathrm{p}<0.05)$ and a subsequent rise $11 \%(\mathrm{p}<0.05)$ of the MAP. This MAP

312 sinusoidal response to SS was not present during the $2^{\text {nd }}, 3^{\text {rd }}, 4^{\text {th }}$ and $5^{\text {th }}$ FLs, while there was a 313 robust and sustained increase in MAP- $\Delta$ peak and AUC (Table 1 and Figure 3). During all the 314 passive knee extension phases (EXs) of SS, MAP rapidly dropped to values similar to 315 baseline (Table 1; Figure 3, panel A). During the $1^{\text {st }} \mathrm{FL}, \mathrm{SV}$ increased by $\sim 22 \mathrm{ml}(\mathrm{p}<0.05$;

316 Figure 3, panel B), and remained significantly elevated from baseline for $\sim 30 \mathrm{~s}$. This SV$317 \Delta$ peak was blunted $(\sim 13 \mathrm{ml})$ during the $2^{\text {nd }}, 3^{\text {rd }}, 4^{\text {th }}$ and $5^{\text {th }} F L s$, while the response was longer 31842 s (Table 1 and Figure 3). During all the passive extension phases (EXs) of SS, SV 319 increased by $\sim 13 \mathrm{ml}$ (Table 1; Figure 3, panel B). During all the FLs phases of SS, both HR 320 and $\mathrm{CO}$ rapidly increased by $\sim 18 \mathrm{BPM}$ and $0-311 \cdot \mathrm{min}^{-1}$ respectively $(\mathrm{p}<0.05$; Figure 3 , 321 panels $\mathrm{C}$ and $\mathrm{D})$. $\mathrm{HR}$ and $\mathrm{CO}$ values remained significantly elevated from baseline for $\sim 42 \mathrm{~s}$.

322 During all the EXs phases of SS, HR and CO rapidly dropped to values similar to baseline 323 (Table 1; Figure 3, panels C and D).

\section{Knee joint range of motion (ROM) and force output (FO) during consecutive bouts of SS}

326 The ROMs and FO attained during the consecutive bouts of SS are reported in Table 2. Both, 327 ROM and FO did not increase from the $1^{\text {st }}$ to the $5^{\text {th }}$ SS bout, and no differences were found 328 in any comparison ( $\mathrm{p}=0.203$ and $\mathrm{p}=0.993$, respectively). 
333 disclosed significant main effects in FBFpeak for Time $(\mathrm{F}=207 ; P<0.001)$ and limb $(\mathrm{F}=$

$3342741 ; P<0.001)$, and a significant time $\mathrm{x} \operatorname{limb}$ interaction $(\mathrm{F}=270 ; P<0.001)$. Similarly,

335 main effects in VC-peaks for factor Time was $(\mathrm{F}=190 ; P<0.001)$ and $\operatorname{limb}(\mathrm{F}=2453 ; P<$

$3360.001)$ and a significant time $\mathrm{x} \operatorname{limb}$ interaction $(\mathrm{F}=243 ; P<0.001)$ were found. In the SL,

337 during the $1^{\text {st }} \mathrm{FL}$ procedure both FBF and VC were transiently increased from the $3^{\text {rd }}$ to the

$33824^{\text {th }}$ second (Figure 4, panels A and B). This initial stretch-induced hyperemic response of SL,

339 in terms of $\Delta$ peak, and AUC, was greater in comparison to the $2^{\text {nd }}, 3^{\text {rd }}, 4^{\text {th }}$ and $5^{\text {th }}$ FLs.

340 Interestingly, a local reduction of FBF and VC was present in the SL from the $3^{\text {rd }}$, to the $5^{\text {th }}$

341 FL. Notably, during all the FL phases of SS, FBF and VC of CL rapidly dropped below the

342 baseline value (Table 2; Figure 4, panels A and B). During the $3^{\text {rd }}, 4^{\text {th }}$ and $5^{\text {th }}$ FL procedures

343 FBF and VC of SL and CL were similar. During all the EX phases of SS, FBF and VC of SL

344 rapidly increased by $\sim 455 \mathrm{ml} / \mathrm{min}$ and $\sim 72 \mathrm{ml} / \mathrm{min} / \mathrm{mmHg}$, respectively (Table 2; Figure 4,

345 panels A and B). While at contrary, FBF and VC of CL rapidly increased to values similar to

346 baseline (Table 2; Figure 4, panels A and B).

\section{Discussion}

349 Although heart and musculoskeletal hemodynamic response to SS have been recently 350 investigated, the role of sympathetic and parasympathetic activity in relation to heart

351 hemodynamic and musculoskeletal circulation in stretched and contralateral muscles has

352 received so far only little attention. In the present study, we investigated the heart

353 hemodynamics and musculoskeletal blood flow responses to repetitive bouts of unilateral SS

354 of the quadriceps muscle. The contribution of the sympathetic and parasympathetic activity to

355 the heart hemodynamics and local difference in the circulation of stretched and contralateral

356 muscles were also investigated. In accordance with our hypothesis, the main findings of the

357 current study were: (i) the heart hemodynamic responses to SS seemed to be primarily 
358 influenced by the parasympathetic withdrawal rather than the increase in sympathetic activity;

359 (ii) the stretch-induced hyperemia in the passively stretched limb was initially greater in 360 comparison to the contralateral limb; and (iii) this local difference in musculoskeletal 361 hemodynamic response to SS was dependent on the repetitive exposures to SS (number of

362 bouts), indicating that after a transiently local hyperemia, a peripheral vasoconstriction 363 occurred presumably triggered by the stretch-induced mechano-reflex.

Interaction between skeletal muscle stretching, autonomic nervous system and central

366 hemodynamics: The present findings advance the knowledge on the interactions between 367 autonomic nervous system and central hemodynamics in the response to SS, during which the 368 sympathetic and parasympathetic activity are partially modulated by the feedbacks that arise 369 from mechano-receptors activation in the skeletal muscle $(1,10,42)$. The data from the 370 current investigation indicate that SS influences the sympatho-vagal balance. However, the 371 rise of the LF/HF index, describing the sympatho-vagal balance of the heart, could occur as a 372 result of an increase of the sympathetic activity, a withdrawal of the vagal tone or a 373 combination of both. Given the decrease of RMSSD, a direct marker of the parasympathetic 374 drive, and the lack of any changes in $\mathrm{LF}_{\mathrm{SBP}}$, a marker of the sympathetic activity, it is 375 reasonable that the increase of $\mathrm{LF} / \mathrm{HF}$ index during SS could be ascribed mainly to the 376 parasympathetic withdrawal in combination with an increase in sympathetic activity (Figure 377 2). Similarly to the blood pressure effect induced by the exercise pressure reflex, the mean 378 DBP rise found in the present study could be likely due to the increases in intramuscular 379 pressure produced during flexion phases of SS (30). In accordance with a previous study (9), 380 such stretch-induced changes in autonomic nervous system discharge were coupled with 381 significant central hemodynamic responses (Table 1, Figure 2), supporting the first hypothesis 382 that not only the cardioacceleration, but also the increase in CO, SV and MAP are primarily 383 influenced by the parasympathetic withdrawal triggered by the mechano-reflex $(9,30)$. 
385 Stretch induced hyper- and hypo-emia in the passively stretched and contralateral limb:

386 From the current study, the high-resolution analysis of peripheral circulation of the passively

387 SL revealed a marked hyperemia in response to the $1^{\text {st }}$ stretching maneuver, which was likely

388 explained by local release of vasoactive substances overcoming sympathetically-mediated

389 vasoconstriction that was likely more relevant at peripheral level in comparison to central

390 level $(37,42)$. Conversely, the concomitant FBF and VC were significantly reduced in the

391 CL. This phenomenon could explain the rise in mean DBP and be likely ascribed to the

392 stretch-induced increases in sympathetic activity, at vascular level, evoked by the mechano-

393 reflex activation triggered by SS in the SL (Table 2 and Figure 4). Indeed, the current data on

394 the stretch-induced hyperemia are in agreement with previous studies that adopted a similar

395 technical approach $(12-14,26,38,42)$. However, this hyperemia in the SL is partially in

396 disagreement with the data reported by a recent study (19), in which the investigators

397 observed no detectable change in net blood flow and VC measured at popliteal artery during 5

398 minutes of SS on the plantar flexors. This discrepancy could be possibly explained by the

399 volume of the stretched muscle, which, in turn, can generate different NO release and greater

400 hyperemia in a larger muscle (42). However, it could be argued that it would be the same

401 when normalized to muscle mass, thus the concentration of NO in the muscle would be

402 comparable resulting in similar impact on vasodilation. Alternatively, this discrepancy may be

403 explained by differences in the magnitude of muscle fiber lengthening. Specifically, a change

404 in joint angle of 90 degrees at the knee and ankle may not yield the same change in muscle

405 fiber length of the muscles that span those joints, due to differences in tendon length and fiber

406 pennation angle. Moreover, in a recent murine study has been demonstrated that passive

407 stretching does not increase NO synthase activity in skeletal muscle (17). Therefore, other

408 physiological mechanisms are potentially involved in this phenomenon. To the best of the

409 authors' knowledge, the current investigation is the first study that have documented a SS- 
induced hypo-emia on a remote muscle not involved in the stretching procedure, therefore a

411 comparison with previous studies is not possible.

412

413 Transitory nature of musculoskeletal stretch-induced hyperemia: From the current study,

414 the analysis of peripheral circulation during consecutive bouts of passive quadriceps muscle

415 SS revealed that the hyperemia in response to the $1^{\text {st }}$ stretching maneuver rapidly disappeared

416 during the $2^{\text {nd }}$ SS procedure. Interestingly, during the $3^{\text {rd }}, 4^{\text {th }}$ and $5^{\text {th }}$ repetitions of SS the

417 blood flow to the stretched muscle was significantly reduced, overlapping that in the CL. This

418 finding suggests that the hyperemia evidenced in the $1^{\text {st }} \mathrm{SS}$ maneuver was likely mediated by

419 local factors, but due to the plausible reduced bioavailability of these factors, the systemic

420 sympathetic-mediated vasoconstriction that was activated by the stretch-induced mechano-

421 reflex prevailed thereafter. Due to the transitory and unstable nature of these local vasoactive

422 factors, such as NO, their direct measurements are rather complicated. In the past NOS

423 inhibitors activators was utilized to understand the role of NO during dynamic passive

424 stretching (37). However, to our knowledge, no studies have investigated the role of NO

425 during SS. In a prospective view, future potential studies could explore the individual

426 contributions of peripheral vasodilators, such as NO, and mechanoreceptor activation on this

427 immediate hyperemic response. For instance, the use of NOS inhibitors activators and afferent

428 blockade in separate or combined stretching trials would be interesting to compare these

429 influences.

Data from the current study indicate no detectable change in the hyperemia of SL

432 during all the five 15-s (EXs) of the SS protocol. Specifically, this constant FBF response

433 during the EX phases could be likely supported by a mechanical reduction in stretch-induced

434 peripheral resistance and the contribution of some metabolic vasodilatory factors released in

435 response to the reduction of venous return following the muscle stretch of the muscle. Indeed, 
SS maneuver likely collapsed the venules and veins thereby reducing venous return. The build

437 of metabolic bioproducts, not due to increased production but rather to decreased clearance,

438 might result in vasodilation and transient hyperemic response observed during the extension

439 phases. These metabolic bioproducts would be quickly washed out and therefore the

440 hyperemic response would be short lived. In a previous investigation (27), it was revealed that

441 FBF was clearly influenced by knee joint angle. In detail, FBF was documented to increase as

442 the knee was extended from the lower $\left(90^{\circ}\right)$ to the middle and upper $\left(0^{\circ}\right.$, full extension) range

443 of knee joint angle. It was concluded that the factors likely involved in this response were

444 muscle length-dependent changes in capillary tortuosity and vessel diameter $(24,33)$. Overall,

445 our data indicate that, in the absence of the local metabolic perturbation, triggered by

446 voluntary exercise, the balance among neural and local factors contributing to the regulation

447 of skeletal muscle blood flow, was likely dependent on the repetitive exposures to SS

448 (number of bouts) and influenced by the reduced bioavailability of local vasoactive factors

449 (i.e., NO) that are released during the initial, passive stretch of the skeletal muscle.

450

\section{Conclusions}

This study documented that heart hemodynamic responses to SS are primarily influenced by the parasympathetic withdrawal rather than the increase in sympathetic activity. The musculoskeletal hemodynamic responses documented in the SL and CL during repetitive exposures to SS, suggest an initial limb-difference in local circulation response to SS, that disappeared during the $3^{\text {rd }}$ repetition of SS. Overall, these results indicate that, the balance among neural and local factors contributing to the regulation of musculoskeletal blood flow, is dependent on the SS exposure suggesting that after a transiently local hyperemia, a systemic sympathetic-mediated vasoconstriction prevailed via the stretch-induced mechanoreflex. 
463 The authors greatly appreciate the time and effort of the subjects that participated to this

464 study. Moreover, we would really thank Prof. Merati Giampiero for his constructive 465 contribution to the revision.

466

467 Grants

$468 \quad$ No funding

469

470 Disclosures

$471 \quad$ None

472

473 


\section{References}

1. Amann M, Sidhu SK, Weavil JC, Mangum TS, and Venturelli M. Autonomic responses to exercise: group III/IV muscle afferents and fatigue. Auton Neurosci 188: 19-23, 2015.

2. Azabji Kenfack M, Lador F, Licker M, Moia C, Tam E, Capelli C, Morel D, and Ferretti G. Cardiac output by Modelflow method from intra-arterial and fingertip pulse pressure profiles. Clinical science 106: 365-369, 2004.

3. Bilchick KC, and Berger RD. Heart rate variability. J Cardiovasc Electrophysiol 17: 691-694, 2006.

4. Bogert LW, and van Lieshout JJ. Non-invasive pulsatile arterial pressure and stroke volume changes from the human finger. Exp Physiol 90: 437-446, 2005.

5. Ce E, Longo S, Rampichini S, Devoto M, Limonta E, Venturelli M, and Esposito F. Stretch-induced changes in tension generation process and stiffness are not accompanied by alterations in muscle architecture of the middle and distal portions of the two gastrocnemii. J Electromyogr Kinesiol 25: 469-478, 2015.

6. Delp MD, and O'Leary DS. Integrative control of the skeletal muscle microcirculation in the maintenance of arterial pressure during exercise. J Appl Physiol (1985) 97: 1112-1118, 2004.

7. Drew RC, Bell MP, and White MJ. Modulation of spontaneous baroreflex control of heart rate and indexes of vagal tone by passive calf muscle stretch during graded metaboreflex activation in humans. J Appl Physiol (1985) 104: 716-723, 2008.

8. Electrophysiology TFotESoCtNASoP. Heart Rate Variability. Circulation 93: 1043, 1996.

9. Farinatti PT, Brandao C, Soares PP, and Duarte AF. Acute effects of stretching exercise on the heart rate variability in subjects with low flexibility levels. Journal of strength and conditioning research / National Strength \& Conditioning Association 25: 1579-1585, 2011.

10. Friedman DB, Jensen FB, Mitchell JH, and Secher NH. Heart rate and arterial blood pressure at the onset of static exercise in man with complete neural blockade. $J$ Physiol 423: 543-550, 1990.

11. Furlan R, Porta A, Costa F, Tank J, Baker L, Schiavi R, Robertson D, Malliani A, and Mosqueda-Garcia R. Oscillatory patterns in sympathetic neural discharge and cardiovascular variables during orthostatic stimulus. Circulation 101: 886-892, 2000.

12. Groot HJ, Rossman MJ, Trinity JD, Layec G, Ives SJ, and Richardson RS. Passive Leg Movement-Induced Vasodilation in Women: The Impact of Age. Am J Physiol Heart Circ Physiol ajpheart 00422 02015, 2015.

13. Groot HJ, Trinity JD, Layec G, Rossman MJ, Ives SJ, Morgan DE, Bledsoe A, and Richardson RS. The role of nitric oxide in passive leg movement-induced vasodilatation with age: insight from alterations in femoral perfusion pressure. J Physiol 2015.

14. Groot HJ, Trinity JD, Layec G, Rossman MJ, Ives SJ, and Richardson RS. Perfusion pressure and movement-induced hyperemia: evidence of limited vascular function and vasodilatory reserve with age. Am J Physiol Heart Circ Physiol 304: H610-619, 2013.

15. Hellsten Y, Nyberg M, Jensen LG, and Mortensen SP. Vasodilator interactions in skeletal muscle blood flow regulation. J Physiol 590: 6297-6305, 2012.

16. Ives SJ, Amann M, Venturelli M, Witman MA, Groot HJ, Wray DW, Morgan DE, Stehlik J, and Richardson RS. The Mechanoreflex and Hemodynamic Response to Passive Leg Movement in Heart Failure. Med Sci Sports Exerc 48: 368-376, 2016.

17. Kerris JP, Betik AC, Li J, and McConell GK. Passive stretch regulates skeletal muscle glucose uptake independent of nitric oxide synthase. J Appl Physiol (1985) 2018. 
18. Kruse NT, and Scheuermann BW. Cardiovascular Responses to Skeletal Muscle Sports Med 47: 2507-2520, 2017.

19. Kruse NT, Silette CR, and Scheuermann BW. Influence of passive stretch on muscle blood flow, oxygenation and central cardiovascular responses in healthy young males. Am J Physiol Heart Circ Physiol 310: H1210-1221, 2016.

20. Limonta E, Rampichini S, Riboli A, Venturelli M, Ce E, and Esposito F. Influence of acute passive stretching on the oxygen uptake vs work rate slope during an incremental cycle test. Eur J Appl Physiol 115: 2583-2592, 2015.

21. Longo S, Devoto M, Monti E, Venturelli M, Limonta E, Rampichini S, Bisconti AV, Esposito F, and Cè E. Acute effects of static stretching on skeletal muscle relaxation at different ankle joint angles. Sport Sciences for Health 12: 429-436, 2016.

22. Loscalzo J, and Welch G. Nitric oxide and its role in the cardiovascular system. Prog Cardiovasc Dis 38: 87-104, 1995.

23. Marchi A, Bari V, De Maria B, Cerutti S, Heusser K, Tank J, Jordan J, Barbic F, Furlan R, and Porta A. Evaluation of the correlation between cardiac and sympathetic baroreflex sensitivity before orthostatic syncope. Conf Proc IEEE Eng Med Biol Soc 2015: 2063-2066, 2015.

24. Mathieu-Costello O. Muscle capillary tortuosity in high altitude mice depends on sarcomere length. Respir Physiol 76: 289-302, 1989.

25. McCormack HM, Horne DJ, and Sheather S. Clinical applications of visual analogue scales: a critical review. Psychol Med 18: 1007-1019, 1988.

26. McDaniel J, Fjeldstad AS, Ives S, Hayman M, Kithas P, and Richardson RS. Central and peripheral contributors to skeletal muscle hyperemia: response to passive limb movement. J Appl Physiol 108: 76-84, 2010.

27. McDaniel J, Ives SJ, and Richardson RS. Human muscle length-dependent changes in blood flow. J Appl Physiol (1985) 112: 560-565, 2012.

28. Merati G, Maggioni MA, Invernizzi PL, Ciapparelli C, Agnello L, Veicsteinas A, and Castiglioni P. Autonomic modulations of heart rate variability and performances in short-distance elite swimmers. Eur J Appl Physiol 115: 825-835, 2015.

29. Mortensen SP, Askew CD, Walker M, Nyberg M, and Hellsten Y. The hyperaemic response to passive leg movement is dependent on nitric oxide: a new tool to evaluate endothelial nitric oxide function. J Physiol 590: 4391-4400, 2012.

30. Murphy MN, Mizuno M, Mitchell JH, and Smith SA. Cardiovascular regulation by skeletal muscle reflexes in health and disease. Am J Physiol Heart Circ Physiol 301: H11911204, 2011.

31. Pagani M, and Malliani A. Interpreting oscillations of muscle sympathetic nerve activity and heart rate variability. J Hypertens 18: 1709-1719, 2000.

32. Pagani M, Montano N, Porta A, Malliani A, Abboud FM, Birkett C, and Somers VK. Relationship between spectral components of cardiovascular variabilities and direct measures of muscle sympathetic nerve activity in humans. Circulation 95: 1441-1448, 1997.

33. Poole DC, Musch TI, and Kindig CA. In vivo microvascular structural and functional consequences of muscle length changes. Am J Physiol 272: H2107-2114, 1997.

34. Sundlof G, and Wallin BG. Human muscle nerve sympathetic activity at rest. Relationship to blood pressure and age. J Physiol 274: 621-637, 1978.

35. Tam E, Azabji Kenfack M, Cautero M, Lador F, Antonutto G, di Prampero PE, Ferretti G, and Capelli C. Correction of cardiac output obtained by Modelflow from finger pulse pressure profiles with a respiratory method in humans. Clinical science 106: 371-376, 2004.

36. Thomas GD, and Segal SS. Neural control of muscle blood flow during exercise. $J$ Appl Physiol (1985) 97: 731-738, 2004. 

Bledsoe A, and Richardson RS. Nitric oxide and passive limb movement: a new approach to assess vascular function. J Physiol 590: 1413-1425, 2012.

578 38. Trinity JD, McDaniel J, Venturelli M, Fjeldstad AS, Ives SJ, Witman MA, 579 Barrett-O'Keefe Z, Amann M, Wray DW, and Richardson RS. Impact of body position 580 on central and peripheral hemodynamic contributions to movement-induced hyperemia: implications for rehabilitative medicine. Am J Physiol Heart Circ Physiol 300: H1885-1891, 2011.

39. VanTeeffelen JW, and Segal SS. Interaction between sympathetic nerve activation and muscle fibre contraction in resistance vessels of hamster retractor muscle. J Physiol 550: 563-574, 2003.

40. Venturelli M, Amann M, Layec G, McDaniel J, Trinity JD, Fjeldstad AS, Ives SJ, Yonnet G, and Richardson RS. Passive leg movement-induced hyperaemia with a spinal cord lesion: evidence of preserved vascular function. Acta Physiol (Oxf) 210: 429-439, 2014. 41. Venturelli M, Amann M, McDaniel J, Trinity JD, Fjeldstad AS, and Richardson RS. Central and peripheral hemodynamic responses to passive limb movement: the role of arousal. Am J Physiol Heart Circ Physiol 302: H333-339, 2012. 42. Venturelli M, Ce E, Limonta E, Bisconti AV, Devoto M, Rampichini S, and Esposito F. Central and peripheral responses to static and dynamic stretch of skeletal muscle: mechano- and metaboreflex implications. J Appl Physiol (1985) 122: 112-120, 2017. 43. Xhyheri B, Manfrini O, Mazzolini M, Pizzi C, and Bugiardini R. Heart rate variability today. Prog Cardiovasc Dis 55: 321-331, 2012.

598 
600 Table 1: Central hemodynamics during the flexion (FL) and extension (EX) phases of

601 consecutive bouts of one leg static stretching (SS).

602

\begin{tabular}{|c|c|c|c|c|c|c|c|}
\hline & & & $1^{\mathrm{st}}$ & $2^{\text {nd }}$ & $3^{\text {rd }}$ & $4^{\text {th }}$ & $5^{\text {th }}$ \\
\hline \multirow{4}{*}{ MAP } & \multirow{2}{*}{$\Delta$ peak $(\mathrm{mmHg})$} & FL & $-7.1 \pm 1.2$ & $14.2 \pm 1.3 \S$ & $11.7 \pm 1.5 \S$ & $10.8 \pm 1.4 \S$ & $11.8 \pm 1.3 \S$ \\
\hline & & EX & $-15.0 \pm 1.3 *$ & $-15.1 \pm 1.4 *$ & $-18.7 \pm 1.7 *$ & $-19.8 \pm 1.6 *$ & $-19.0 \pm 1.8 *$ \\
\hline & \multirow{2}{*}{$\mathrm{AUC}(\mathrm{mmHg} \cdot \mathrm{s})$} & FL & $0.01 \pm 0.01$ & $7.5 \pm 0.03 \S$ & $6.6 \pm 0.09 \S$ & $6.9 \pm 0.07 \S$ & $7.6 \pm 0.05 \S$ \\
\hline & & EX & $-2.08 \pm 0.01 *$ & $-2.58 \pm 0.02 *$ & $-2.25 \pm 0.02 *$ & $-2.56 \pm 0.02 *$ & $-2.88 \pm 0.03 *$ \\
\hline \multirow{4}{*}{ SV } & \multirow{2}{*}{$\Delta$ peak (ml) } & FL & $21.8 \pm 2.4$ & $13.6 \pm 2.5 \S$ & $14.3 \pm 1.9 \S$ & $12.1 \pm 1.9 \S$ & $13.0 \pm 2.2 \S$ \\
\hline & & EX & $16.7 \pm 2.5$ & $9.0 \pm 2.2 \S$ & $8.1 \pm 2.9 \S$ & $10.2 \pm 1.6 \S$ & $8.0 \pm 3.2 \S$ \\
\hline & \multirow{2}{*}{$\operatorname{AUC}(\mathrm{ml} \cdot \mathrm{s})$} & FL & $10.6 \pm 0.32$ & $8.3 \pm 0.28$ & $8.9 \pm 0.22$ & $7.9 \pm 0.22$ & $7.1 \pm 0.24$ \\
\hline & & EX & $1.2 \pm 0.02 *$ & $0.5 \pm 0.08 * \S$ & $0.4 \pm 0.02 * \S$ & $0.6 \pm 0.02 * \S$ & $0.7 \pm 0.04 * \S$ \\
\hline \multirow{4}{*}{ HR } & \multirow{2}{*}{$\Delta$ peak (BPM) } & FL & $18 \pm 1.9$ & $16 \pm 1.9$ & $18 \pm 2.0$ & $20 \pm 1.8$ & $20 \pm 2.8$ \\
\hline & & EX & $-16 \pm 2.1 *$ & $-16 \pm 2.3 *$ & $-18 \pm 2.9 *$ & $-18 \pm 2.9 *$ & $-18 \pm 3.8 *$ \\
\hline & \multirow{2}{*}{ AUC (beats) } & FL & $11.5 \pm 0.9$ & $8.5 \pm 0.8$ & $11.3 \pm 0.9$ & $12.2 \pm 0.82$ & $12.9 \pm 0.9$ \\
\hline & & EX & $-2.46 \pm 0.11 *$ & $-2.91 \pm 0.10 *$ & $-3.35 \pm 0.10 *$ & $-3.36 \pm 0.11 *$ & $-3.25 \pm 0.09 *$ \\
\hline \multirow{4}{*}{$\mathrm{CO}$} & \multirow{2}{*}{$\Delta$ peak $\left(1 \cdot \mathrm{min}^{-1}\right)$} & FL & $0.32 \pm 0.08$ & $0.26 \pm 0.07$ & $0.25 \pm 0.09$ & $0.35 \pm 0.09$ & $0.27 \pm 0.10$ \\
\hline & & EX & $-0.16 \pm 0.07 *$ & $-0.30 \pm 0.05 *$ & $-0.28 \pm 0.07 *$ & $-0.26 \pm 0.08 *$ & $-0.31 \pm 0.09 *$ \\
\hline & \multirow{2}{*}{ AUC (1) } & FL & $0.14 \pm 0.02$ & $0.16 \pm 0.03$ & $0.15 \pm 0.03$ & $0.21 \pm 0.02$ & $0.15 \pm 0.02$ \\
\hline & & EX & $-0.03 \pm 0.01 *$ & $-0.04 \pm 0.01 *$ & $-0.04 \pm 0.01 *$ & $-0.04 \pm 0.01 *$ & $-0.05 \pm 0.02 *$ \\
\hline
\end{tabular}

603

$604 \Delta$ peak, absolute change; AUC, area under the curve. Cardiac output (CO), stroke volume 605 (SV), heart rate (HR), mean arterial pressure (MAP),

$606 *=\mathrm{p}<0.05$ from FL; $\S=\mathrm{p}<0.05$ from $1^{\text {st }}$. Data are presented as mean $\pm \mathrm{SEM}$.

607

608 
610 Table 2: Knee-joint angle, force output and peripheral hemodynamics during the flexion (FL)

611 and extension (EX) phases of consecutive bouts of one leg static stretching (SS).

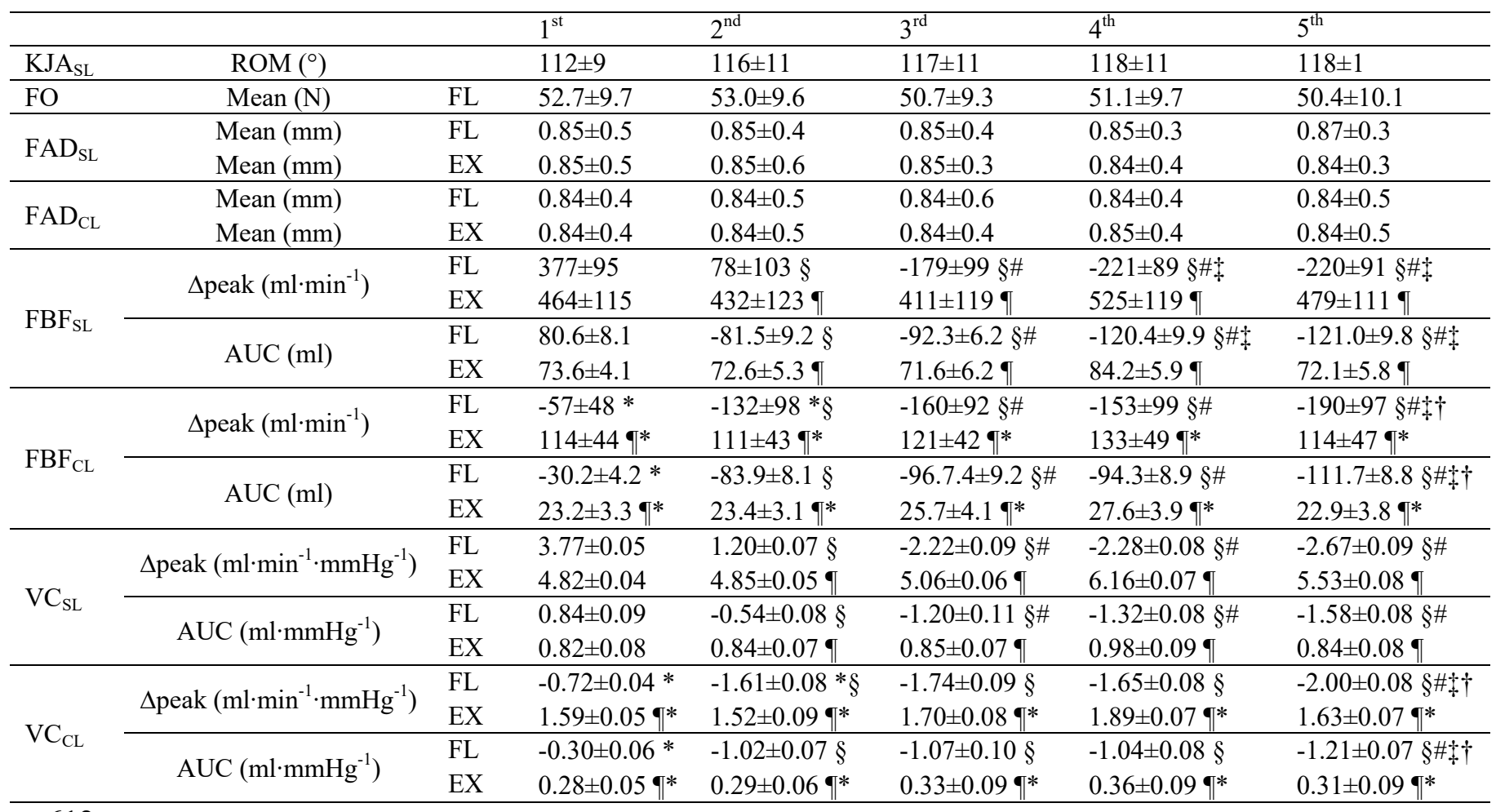

612

613 ROM, range of motion; FO, force output; $\Delta$ peak, absolute change; AUC, area under the 614 curve. Knee-joint angle in stretch leg $\left(\mathrm{KJA}_{\mathrm{SL}}\right)$, Femoral artery diameter in stretched leg $615\left(\mathrm{FAD}_{\mathrm{SL}}\right)$, Femoral artery diameter in control leg $\left(\mathrm{FAD}_{\mathrm{CL}}\right)$, Femoral blood flow in stretch leg $616\left(\mathrm{FBF}_{\mathrm{SL}}\right)$, femoral blood flow in control leg $\left(\mathrm{FBF}_{\mathrm{CL}}\right)$, vascular conductance in stretch leg $617 \quad\left(\mathrm{VC}_{\mathrm{SL}}\right)$, vascular conductance in control leg $\left(\mathrm{VC}_{\mathrm{CL}}\right)$.

$618 \mathrm{q}=\mathrm{p}<0.05$ from FL; $*=\mathrm{p}<0.05$ from stretch leg; $\S=\mathrm{p}<0.05$ from $1^{\text {st }} ; \#=\mathrm{p}<0.05$ from $6192^{\text {nd }} ;+=\mathrm{p}<0.05$ from $3^{\text {rd }} ; \dagger=\mathrm{p}<0.05$ from $4^{\text {th }}$. Data are presented as mean \pm SEM.

620 
Figure 1: Schematic figure showing the position of the subject during the SS procedure. Stretched leg (SL) and control resting leg (CL), range of motion (ROM).

625

626

627

628

629

630

631

632

633

634

635

636

637

638

639

640

641

642

643

644

645

646

647

648

Figure 2: Sympathetic and parasympathetic indexes during baseline and passive static stretching. Panel $A, B, C$ and $D$, illustrate respectively the root mean square of the squared differences of successive NN intervals (RMSSD), the ratio between low and high frequency (LF/HF) of the heart rate variability (HRV), mean diastolic blood pressure (DBP), and the low frequency component of systolic $\left(\mathrm{LF}_{\mathrm{SBP}}\right)$ blood pressure (BPV). Data are mean $\pm \mathrm{SEM} ; *$ significantly different from baseline.

Figure 3: Changes in central hemodynamic responses to repetitive bouts of one leg static stretching. Panels A, B, C, and D illustrate mean arterial pressure (MAP), stroke volume (SV), heart rate (HR), and cardiac output (CO), at baseline (BL), during knee static passive flexion (FL) and static passive extension (EX) respectively. Data are mean \pm $\mathrm{SEM} ; *$ significantly increased from baseline (BL); $\uparrow$ significantly reduced from baseline.

Figure 4: Changes in femoral blood flow and vascular conductance in the stretched leg (SL) and control resting leg (CL) to 5 sequences of SS, at baseline (BL), during knee static passive flexion (FL) and static passive extension (EX) respectively. Data are mean \pm $\mathrm{SEM} ; *$ significantly increased from baseline (BL); $\uparrow$ significantly reduced from baseline; and gray areas indicate significantly different values between SL and CL. 


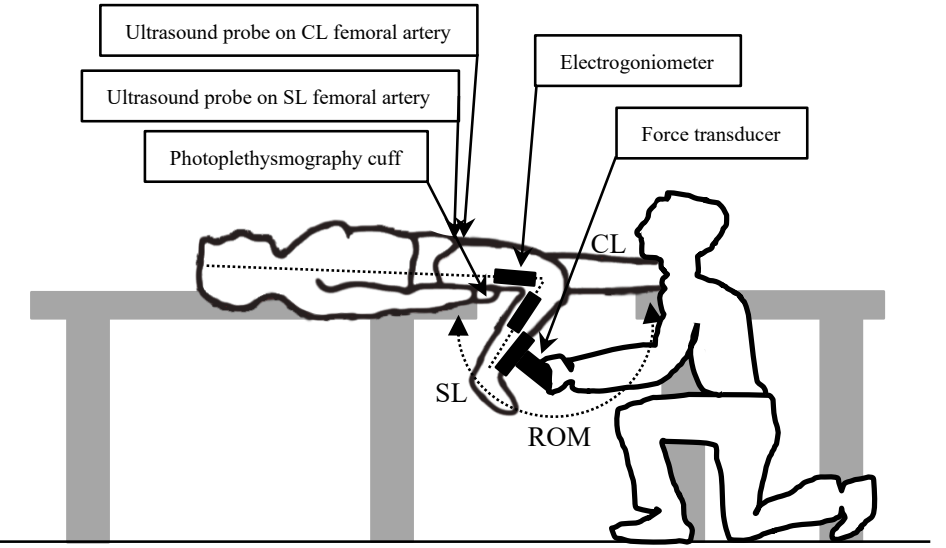

m www.physiology.org/journal/jappl at Biblioteca Polo Didattico (159.149.103.009) on Fig 1 


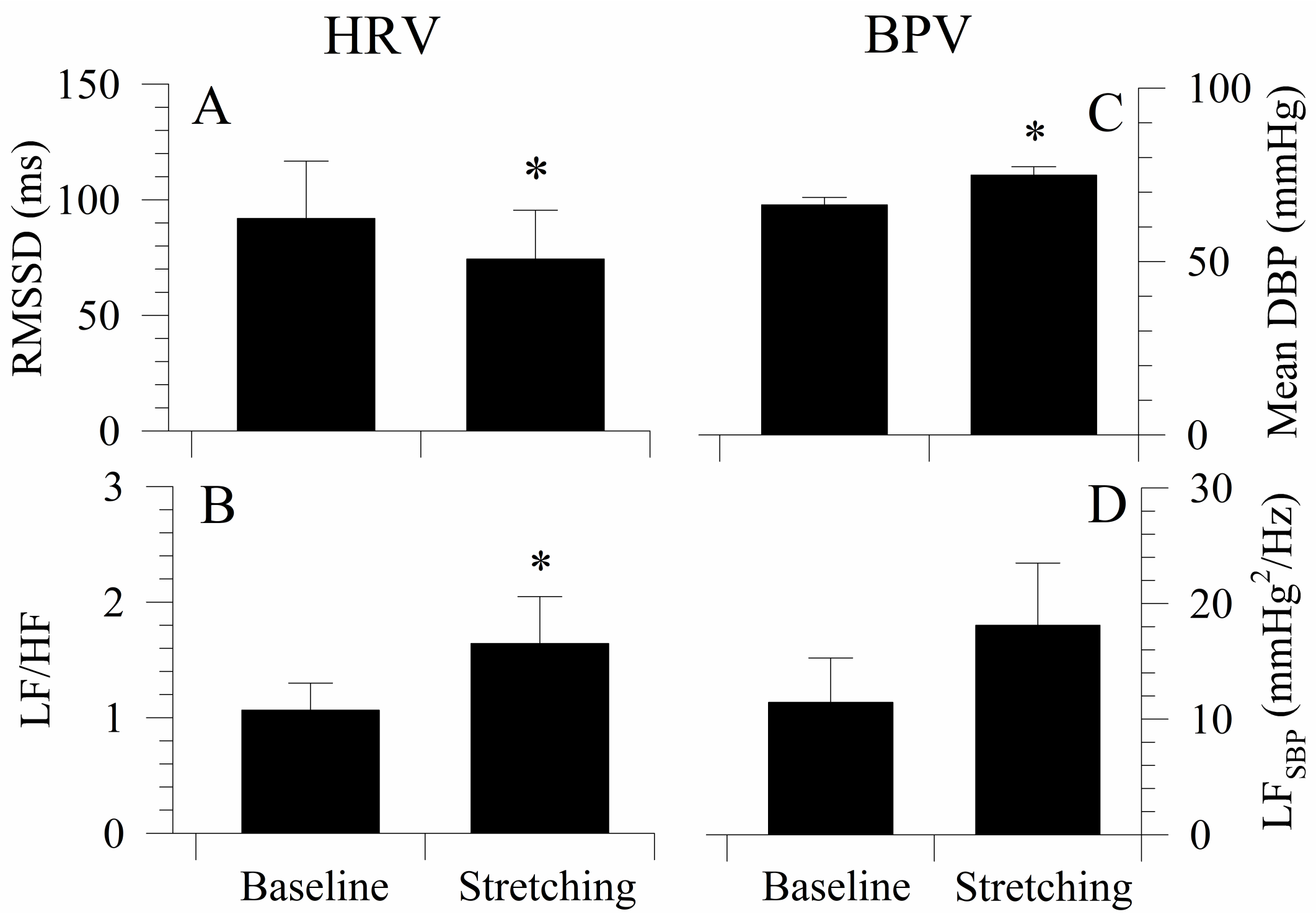




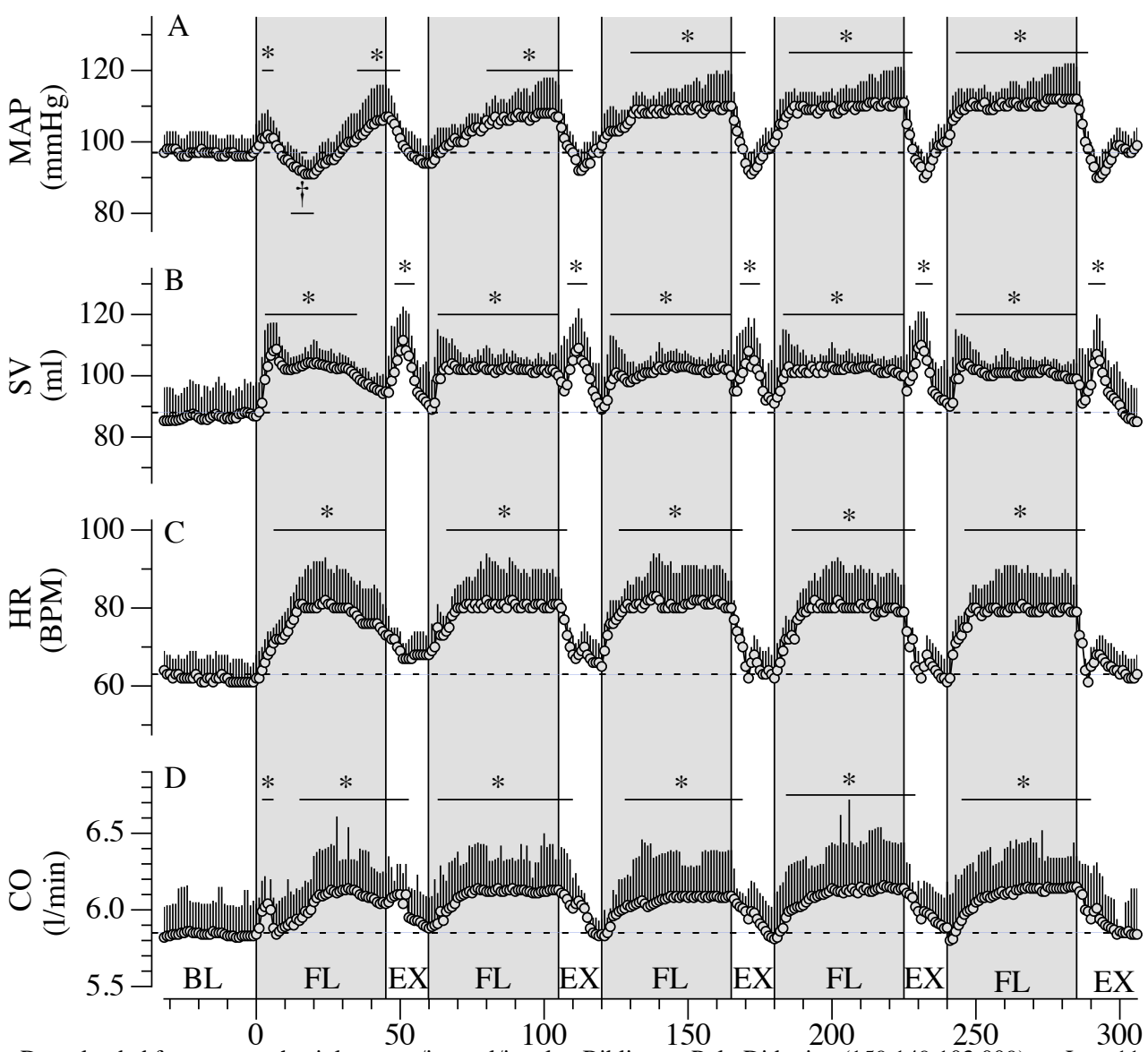

Downloaded from www.physiology.org/journal/jappl at Biblioteca Polo Didattico (159.149.103.009) on June $11,2019$. Figure 3 Time (s) 


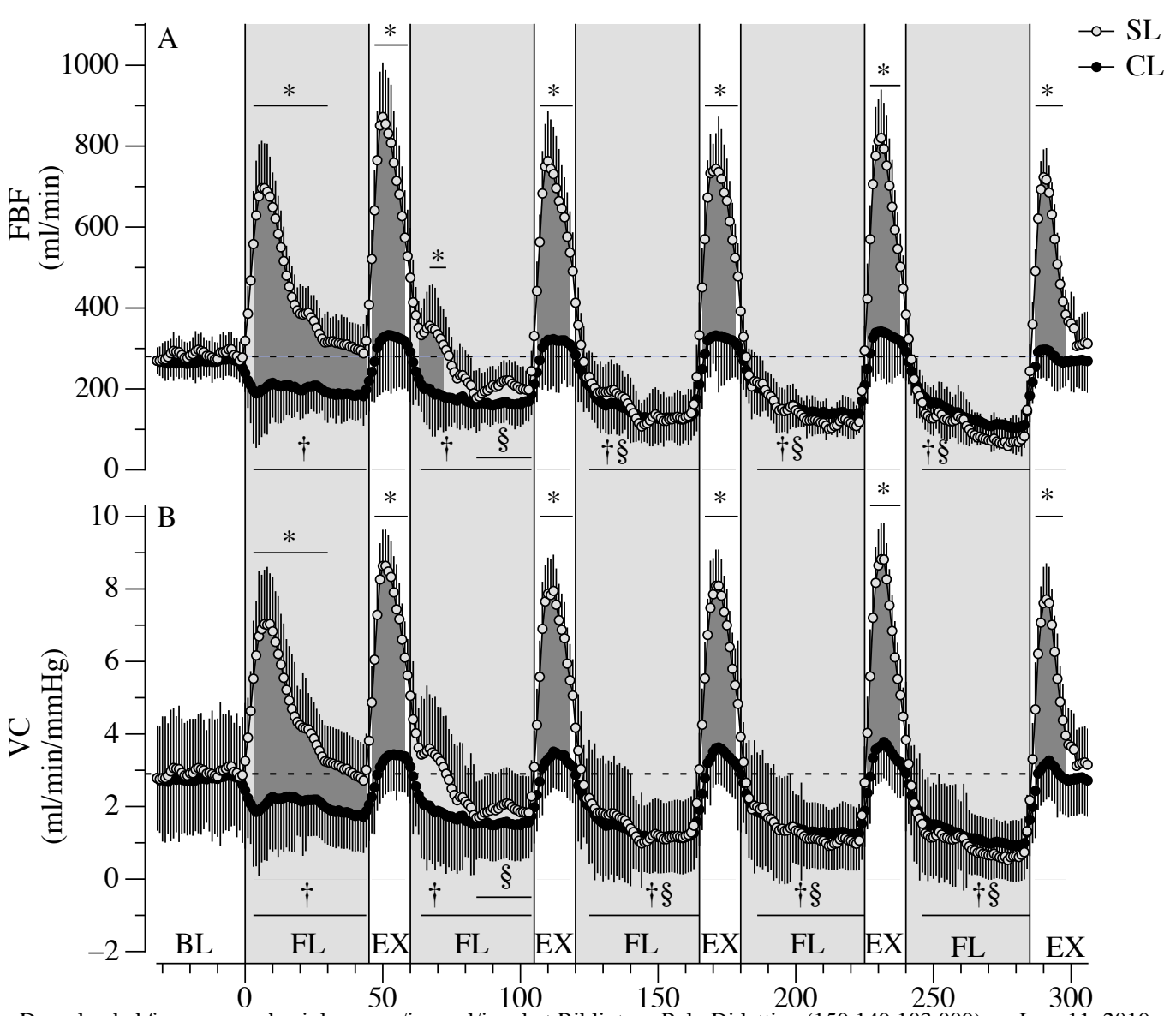

Downloaded from www.physiology.org/journal/jappl at Biblioteca Polo Didattico (159.149.103.009) on June 11, 2019. Figure 4 Time (s) 